\title{
El acoso laboral o mobbing como elemento causante de accidentes laborales
}

\section{Resumen}

El análisis de la influencia del mobbing, como elemento causante de accidentes laborales, es el objetivo general del presente trabajo, resaltando la problemática existente en el desarrollo de riesgos psicosociales que afectan emocional, física y psicológicamente a los trabajadores hoy en día. Para ello, se analizaron distintos autores expertos, así como las normas jurídicas de la Constitución de la República Bolivariana de Venezuela, la Lopcymat, su reglamento, la Constitución de la República del Ecuador, Código del Trabajo Ecuatoriano y el Código Penal Ecuatoriano. El tipo de investigación fue documental con un diseño bibliográfico, la población estuvo constituida por la doctrina y leyes. Asimismo, se utilizó la técnica de observación documental, ya que se efectuó una revisión y análisis de fuentes documentales, así como de contenido. Como resultado, se obtuvo que el mobbing es uno de los más graves factores de riesgos psicosocial, ya que en este proceso se ocasionan graves daños físicos, emocionales y psicológicos en el trabajador acosado de manera intencional y deliberada. Además, se concluyó que el psicoterror deja a sus víctimas en un estado de permanente hipervigilancia, lo que finalmente le ocasiona un estado de estrés intenso, y una vez agotado queda susceptible no solo a sufrir un accidente de trabajo, sino también podría llegar a suicidarse.

Palabras clave: mobbing, acoso laboral, psicoterror, riesgos psicosociales.

\section{Abstract}


The aim of this article is to analyze the influence of mobbing as an element that causes occupational accidents. This problem affects the development of psychosocial risks that affect workers emotionally, physically and psychologically. To this end, several authors were analyzed, as well as the legal laws of the Constitution of the Bolivarian Republic of Venezuela, the Lopcymat, its regulations, the Constitution of the Republic of Ecuador, the Ecuadorian Labor Code and the Ecuadorian Penal Code. The type of research was documentary with a bibliographic design, the population was constituted by doctrine and laws. The documentary observation technique was also used, as well as a review and analysis of the bibliography. As a result, mobbing is one of the most serious factors of psychosocial risks, since serious physical, emotional and psychological damages are caused in the worker being harassed intentionally and deliberately. It was also concluded that mobbing leaves its victims in a state of permanent hypervigilance, eventually causing a state of intense stress, leaving the person sensitive not only to a work accident but could also commit suicide.

Keywords: mobbing, occupational harassment, psychoterror, psychosocial risks. 


\section{Introducción}

Actualmente las relaciones de trabajo suelen verse afligidas por conductas desviadas de los miembros de un grupo de trabajo, incidiendo negativamente en las condiciones bajo las cuales las personas pertenecientes a dicho grupo deben prestar sus servicios, además de afectar el clima laboral, aunado a una mala organización del trabajo, recae en la conducta del trabajador, dando lugar a los denominados riesgos psicosociales, principalmente los relacionados a la violencia en el lugar de trabajo y, más concretamente, con el mobbing o acoso psicológico en el trabajo.

La violencia psicológica causa estragos a la salud emocional e impide el disfrute de un ambiente sano y saludable en el que los individuos puedan desenvolverse y desarrollar una mejor calidad de vida. Recientemente, la violencia psicológica ha traído una consecuencia especifica en el ámbito laboral, ha hecho surgir un problema psicológico con graves efectos en todos los aspectos de la vida de la persona afectada, este problema psicológico se ha ido expandiendo poco a poco y silenciosamente sin conseguir un mayor número de denuncias legales. El acoso moral, acoso psicológico o psicoterror, se ha hecho cada vez más notorio afectando un mayor número de personas.

El acoso moral o en su anglicismo mobbing, se refiere a acciones negativas recurrentes que se manifiestan a través de comportamientos, palabras, actos y gestos hostiles no deseados, dirigido a una o varias personas, atentando contra su personalidad, dignidad, integridad física o psíquica; con el firme propósito de que esta persona abandone su lugar de trabajo después de que se ha degradado su ambiente laboral, se han destruido sus redes de comunicación, su reputación, el ejercicio de sus labores y sus relaciones sociales.

Tomando en cuenta lo argumentado, la Organización Internacional de Trabajo (OIT) sostiene que prácticas como la intimidación, hostigamiento sistemático o mobbing han aumentado, de tal manera que ha alcanzado niveles considerados como epidemia, lo cual puede llegar a ser tan dañino como la violencia física. En este contexto, el psicoterror resulta contrario a lo expuesto en la Declaración Universal de los Derechos Humanos (DUDH), que, entre otros aspectos, se estará explicando en el presente trabajo investigativo.

\section{La problemática del mobbing}

Con el firme propósito de fundamentar la información necesaria para el análisis del mobbing, como elemento causante de accidentes de trabajo, se presenta un breve, pero conciso sustento teórico doctrinal, que tiene la finalidad de presentar la problemática que motiva el desarrollo de este estudio.

Hablar de organización del trabajo supone pensar en un grupo de individuos que unen sus esfuerzos por lograr una meta común, para alcanzar este fin llegan a tener una convivencia de un sin número de horas, lo cual los convierte además en un grupo social dentro del que cada persona cumple una función específica que lo relaciona al resto del grupo, de una determinada manera y que, por último, en sincronía, estas diversas funciones y relaciones suponen el alcance del objetivo común. 
Sin embargo, en ocasiones suelen presentarse ciertas conductas o actitudes negativas que atentan contra el desarrollo armónico de las relaciones de trabajo entre los miembros de una organización, con lo cual se genera una situación de incertidumbre que deriva en un deterioro del clima laboral y en condiciones de trabajo inadecuadas, ello a su vez se traduce en una disminución del rendimiento del trabajador, por cuanto se afecta su salud o bienestar psicológico.

Es así como profundos cambios sucedidos en la organización del trabajo, generados por una intensificación del mismo o simplemente por diferencias entre seres humanos, han supuesto la aparición de nuevos riesgos psicosociales que no por intangibles son menos peligrosos.

Una mala organización influye tanto en la salud, como en la conducta del trabajador, dando lugar a los denominados riesgos psicosociales, entre los que se encuentran los relacionados con la violencia física o psicológica y, más concretamente, con el mobbing, acoso moral o psicológico en el trabajo. Según Barbado, P. (2004), aunque las causas de este fenómeno puedan ser de distinta naturaleza, el "mobbing", acoso moral o psicológico somete a la víctima a un proceso sistemático de estigmatización y privación de sus derechos humanos al mantener esta dinámica por un periodo prolongado de tiempo e incluso años, se produce en la victima importantes trastornos en la salud, incluyendo consecuencias físicas y psicológicas que en definitiva excluyen del mercado laboral a la víctima.

Este problema laboral se puede llegar a presentar entre los integrantes de una empresa o institución; a saber, entre el empleador y un trabajador, en un grupo de trabajadores entre sí, ya sea por parte de colegas, superiores o inferiores jerárquicos. Toda esta presión ejercida sin importar su direccionalidad influye en el medio ambiente laboral, trayendo consecuencias post-traumáticas en el trabajador acosado moralmente, pues los efectos del mobbing son tan devastadores que buscan limar, reducir y, en última instancia, destrozar los sentimientos más profundos de la víctima.

Aunado a esto, Barbado, P. (2004) manifiesta que el acoso moral, a diferencia de otros tipos de violencias que pueden presentarse en el ambiente de trabajo, no deja rastros ni señal externa alguna en la victima, salvo por un deterioro progresivo que incide negativamente sobre la salud física y psíquica, ocasionando patologías graves como depresión, trastornos de ansiedad, insomnios, entre otros, que en definitiva crea en el acosado un estado de inestabilidad que lo hace propenso a sufrir lesiones dentro y fuera del lugar de trabajo.

Adicionalmente, Guevara, L. (2003) sostiene que el mobbing se evidencia cuando un individuo, ejerciendo su poder, rebasa los límites y comienza a causar daños a otra persona, dejando de pensar en esta última como ser humano para considerarla entonces un objeto susceptible de dominación, todo esto atenta contra la integridad moral de la víctima, generando en ella alteraciones psicológicas que, según las circunstancias, pueden convertirse en alteraciones patológicas o lesiones. Asimismo, el mobbing afecta la integridad de los trabajadores y su dignidad humana, su calidad de vida, condiciones, seguridad y salud del trabajo.

Tomando en cuenta lo antes planteado, el derecho debe tutelar tanto la integridad física, como la moral de los individuos, ya que el hombre es una unidad biopsicosocial en la que influyen

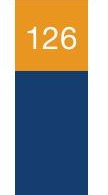


diferentes factores y en caso de acoso moral, el daño primeramente es psíquico y pasa a ser un daño físico cuando el acosado moralmente se ve imposibilitado a seguir confrontando el acoso, y ya cansado de manipulaciones, se ocasiona lesiones dentro o fuera del lugar de trabajo que pueden llegar incluso a representar su muerte o suicidio.

Por otra parte, la OIT (2006) declaró que prácticas como la intimidación, hostigamiento sistemático o mobbing han ido aumentando mundialmente, alcanzando niveles de epidemia, afirmando que el acoso y otras conductas similares pueden ser tan dañinas como la pura y simple violencia física, trayendo como consecuencia la inestabilidad de muchos tipos de empleos, generando enormes presiones en el lugar de trabajo, contribuyendo así con la ya volátil situación generada por una mezcla de agresiones.

Bajo este contexto, el psicoterror resulta contrario a lo expuesto en la Declaración Universal de los Derechos Humanos, en la cual se establece el derecho que tiene toda persona a condiciones equitativas y satisfactorias de trabajo.

\section{El acoso o mobbing en la legislación venezolana}

La Constitución de la República Bolivariana de Venezuela establece en el artículo 3 que el estado tiene como fines esenciales la defensa y el desarrollo de la persona y el respeto a su dignidad, el ejercicio democrático de la voluntad popular, la construcción de una sociedad justa y amante de la paz, la promoción de la prosperidad y bienestar del pueblo y la garantía del cumplimiento de los principios, derechos y deberes consagrados en esta Constitución.

Sobre la validez de lo antes expuesto, toda legislación, buscando el bienestar biopsicosocial del ciudadano, crea parámetros bajo los cuales se regulen los diversos aspectos de la vida civil, social e incluso laboral, es así como en Venezuela la Ley Orgánica del Trabajo Trabajadores y Trabajadoras en su Artículo 1 expresa: "Esta Ley tiene por objeto proteger al trabajo como hecho social y garantizar los derechos de los trabajadores y de las trabajadoras, creadores de la riqueza socialmente producida y sujetos protagónicos de los procesos de educación y trabajo para alcanzar los fines del Estado (...)"

Volviendo la mirada a lo dañino que resulta el mobbing para cualquier persona, es importante determinar los efectos primarios que este hecho puede generar en un individuo, pero más importante aún, establecer aquellas consecuencias que parten de los primeros daños causados, es así como este estudio busca determinar como el mobbing pudiese hacer a la víctima más propensa a cualquier accidente de trabajo.

La Ley Orgánica de Prevención, Condiciones y Medio Ambiente de Trabajo (LOPCYMAT) define los accidentes de trabajo como todo suceso que produce en el trabajador o la trabajadora una lesión funcional o corporal, permanente o temporal, inmediata o posterior, o la muerte, resultante de una acción que pueda ser determinada o sobrevenida en el curso del trabajo, por el hecho o con ocasión del trabajo. 
Sin embargo, a lo largo de la LOPCYMAT y su reglamento no se menciona explícitamente el mobbing o acoso moral como posible causa de los ya mencionados accidentes de trabajo, y a su violación al derecho a la dignidad, intimidad e igualdad de trato, hecho que no solo crea un vacío legal a este respecto, sino que además no establece medidas exhaustivas y sistemáticas para prevenir, corregir y sancionar el acoso moral en el ámbito laboral.

Contrariamente a esta situación, en lo establecido por Velásquez, M. (2002) expresa que los daños y lesiones causadas por el mobbing pueden considerarse como accidentes de trabajo o enfermedad profesional ya que, al hostigar al trabajador acosado, provocando su humillación y definitivo alejamiento del lugar de trabajo, le ocasiona un daño psicológico que afecta su salud, como consecuencia de una persecución de tipo psicológica que va más allá de una simple vulneración de derechos laborales.

De cualquier forma, las citadas autoras Barbado, P. (2004) y Guevara, L. (2003) coinciden que es evidente que el acoso moral representa la posibilidad de que un trabajador sufra un determinado daño derivado del trabajo como enfermedades, patologías o lesiones, lo que provoca graves consecuencias sobre la calidad de vida, las condiciones de trabajo y la salud de los trabajadores que lo padecen, afectando su integridad física y psicológica, pudiendo el mobbing llegar a ser en principio considerado un elemento causante de accidentes laborales.

Con lo anterior expuesto, se puede inferir la necesidad emergente de analizar el mobbing como elemento causante de accidentes laborales, ya que la violencia es un acto contra el ejercicio de la dignidad y la integridad moral de la persona. Por tanto, atenta contra los derechos fundamentales del hombre reconocidos en la Declaración Universal de los Derechos Humanos de 1948, otros Pactos y Declaraciones Internacionales, las Constituciones y otras leyes. La dignidad e igualdad de los trabajadores en el puesto de trabajo es el bien protegido.

\section{El acoso o mobbing en la legislación ecuatoriana}

Las conductas que se denuncien como acoso laboral deben ser valoradas por la autoridad de trabajo, según las circunstancias del caso, y la gravedad de las conductas denunciadas. La autoridad competente apreciará las circunstancias de acuerdo a la capacidad de estas de someter a un trabajador a presión para provocar su marginación, renuncia o abandono de su puesto.

Es obligación del empleador, tal como se lo establece en el numeral 36 del artículo 42, del Código del Trabajo, implementar programas de capacitación y políticas orientadas a identificar las distintas modalidades del acoso laboral para prevenir el cometimiento de toda forma de discriminación, hostigamiento, intimidación y perturbación que se pudiera generar en la relación laboral con los trabajadores y de éstos con el empleador. Le está prohibido al empleador, tal como lo establece el literal "m", del artículo 44 ibidem, el cometimiento de actos de acoso laboral o la autorización de los mismos, por acción u omisión

Tal como lo determina el literal "j" del artículo 46, de Código del Trabajo, le está prohibido al trabajador, el cometimiento de actos de acoso laboral hacia un compañero o compañera, hacia el empleador, hacia un superior jerárquico o hacia una persona subordinada en la empresa.




En virtud de lo estipulado en el numeral octavo del artículo 172 del Código del Trabajo, el trabajador puede ser sujeto de visto bueno por el cometimiento de acoso laboral, ya sea de manera individual o coordinada con otros individuos, hacia un compañero o compañera de trabajo, hacia el empleador o empleadora o hacia un subordinado o subordinada en la empresa. A su vez, tal como lo determina el numeral cuarto del artículo 173 del Código de Trabajo, el trabajador puede solicitar el respectivo visto bueno en casos de sufrir acoso laboral, cometido o permitido por acción u omisión por el empleador o empleadora o sus representantes legales. Previa a la petición del visto bueno, es obligación la apertura de una conciliación que será presidida por la autoridad laboral competente, en la que serán oídos, además del interesado, los representantes de los trabajadores y el empleador o quien le represente.

\subsection{Sanciones}

El trabajador que ha sido víctima de acoso laboral recibirá la indemnización equivalente al valor de un año de la remuneración que venía percibiendo, además de la general que corresponda por despido intempestivo. Atendiendo a la gravedad del caso, la víctima de acoso puede solicitar ante la autoridad laboral competente la disculpa pública de quien cometió la conducta, tal como lo determina el numeral octavo del artículo 545 del Código del Trabajo. Cuando el trabajador o trabajadora presente indicios fundados de haber sufrido acoso laboral le corresponde al empleador o empleadora presentar una justificación objetiva y razonable, suficientemente probada, de las medidas adoptadas y de su proporcionalidad.

Pese a esto, en el Código Penal en vigor no se tipifica una serie de conductas delictivas que surgen en las relaciones de trabajo, cuyos actos afectan a bienes jurídicos de especial significación social, produciendo consecuencias físicas y psíquicas que generan cuadros de ansiedad, dolor, inestabilidad, depresión y otros estados de ánimo que afectan la integridad personal de los trabajadores. Por esto, se recomienda la introducción de una reforma legal que llene este vacío existente en nuestro orden jurídico penal mediante nuevas normas jurídicas que tipifiquen el delito de acoso laboral, las mismas que deberán constar en el Capítulo VIII.1, bajo la denominación: "el ilícito laboral en las relaciones de trabajo", y para ello, someto a consideración de la asamblea la presente iniciativa, a fin de que sea acogida y se convierta un proyecto de ley que beneficie a un sinnúmero de trabajadores que sufren acoso laboral en sus lugares de trabajo.

\section{Tipos de mobbing}

Cuando se analizan los tipos de mobbing según Hirigoyen (2001) citando a Leymann, nos dice que, dependiendo de la posición jerárquica, el mobbing puede ser:

Mobbing horizontal: este se caracteriza porque el acosador y la víctima se encuentran en el mismo rango jerárquico, es decir, que suele darse entre compañeros de trabajo, y las repercusiones a nivel psicológico para la víctima pueden ser devastadoras.

Las causas de este tipo de acoso laboral pueden ser muchas y variadas, aunque las más comunes son para forzar a un trabajador a conformarse con determinadas normas, por enemistad, para 
atacar al más débil, por las diferencias con la víctima, o por falta de trabajo y el aburrimiento.

Mobbing vertical: recibe este nombre porque o bien el acosador se encuentra en un nivel jerárquico superior a la víctima, o se encuentra en un nivel inferior a ésta. Por tanto, existen dos clases de mobbing vertical: ascendente y descendente.

- Mobbing ascendente: ocurre cuando un empleado de nivel jerárquico superior es atacado por uno o varios de sus subordinados.

- Mobbing descendente o bossing: ocurre cuando un empleado de nivel jerárquico inferior recibe acoso psicológico por parte del uno o varios empleados que ocupan posiciones superiores en la jerarquía de la empresa.

\section{Metodología}

El presente estudio es de tipo documental, ya que la información se obtuvo de fuentes documentales, elaborado sobre la base de documentos o revisión bibliográfica y los diseños de modelos propuestos.

Pelekais, Finol, Neuman y Parada (2005) describen al diseño de la investigación como el plan de acción que indica la secuencia de pasos a seguir, permitiendo al investigador precisar detalles de la tarea de investigación y establecer las estrategias a seguir para obtener resultados positivos, además de definir la forma de encontrar respuestas a las interrogantes que inducen el estudio

Con respecto a los instrumentos utilizados se encuentran documentos de diversas índoles, tanto bibliográficos, electrónicos como legales, referentes al tema del mobbing como elemento causante de accidentes de trabajo.

Las técnicas más comunes en las ciencias sociales son las relacionadas con la observación, el análisis de contenido, diferencial semántica, el análisis lógico y el análisis ideológico para la investigación documental. De esta manera, los datos son recolectados realizando una organización, clasificación y análisis previo, de acuerdo al análisis documental del contenido semántico, gramatical e histórico, de acuerdo a un análisis crítico comparativo.

\section{Resultados}

Volviendo a lo expuesto en el planteamiento del problema cabría preguntarse ¿Qué influencia tiene el mobbing en los accidentes de trabajos?, de esta interrogante se derivan una serie de inquietudes que ayudarán a determinar en esta investigación si el mobbing tiene influencia o no en los accidentes de trabajo, estas inquietudes son ¿por qué se considera el mobbing un factor de riesgo psicosocial en las relaciones laborales?, ¿cuáles son los efectos del mobbing en el trabajador acosado moralmente? y ¿qué elementos del mobbing generan accidentes laborales?

Para tal efecto, y con basamento en las interrogantes expuestas, se plantea la inminente

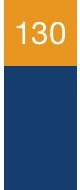


necesidad de realizar un análisis que permita verificar al mobbing como elemento causante de accidentes de trabajo.

Heinz Leymann (1996) utilizó el término mobbing para referirse a aquella situación en la que el individuo, de forma individual o colectiva, ejecuta una violencia psicológica extrema de manera sistemática durante un periodo de tiempo prolongado sobre otra persona o personas en el lugar de trabajo. La principal característica de este acoso radica en su carácter de violencia psicológica (manipulación de la comunicación, reputación, del trabajo y un trato laboral desigual y discriminatorio), ejercida de manera reiterada y prolongada en el tiempo, con la intencionalidad de sacar a la víctima de su lugar de trabajo, lo cual termina teniendo graves efectos en la persona afectada.

González, M. (2005) considera al acoso moral como algo de extrema gravedad calificándolo como un verdadero síndrome, el cual es definido como un conjunto de signos y síntomas que padece la víctima del acoso, que lo pueden llevar al desarrollo de diversas patologías psicosomáticas afectando todos los niveles y ámbitos de la vida del acosado incluidos accidentes y el suicidio.

De esta manera, el autor plantea que esta patología, que se desarrolla en forma encubierta y desconocida, es necesaria desenmascararla para que la víctima identifique rápidamente cuáles son las razones que está sufriendo y pueda sin culpa solicitar la ayuda profesional que permita un tratamiento integral de la problemática.

A modo de ejemplo, Velázquez, M (2001) expresa un pronunciamiento de la Audiencia Provincial de Madrid donde describió en su momento algunas conductas que pueden llegar a darse: "1) ataques mediante medidas adoptadas contra la víctima: el superior le limita las posibilidades de comunicarse, le cambia la ubicación separándole de sus compañeros, se juzga de manera ofensiva su trabajo, se cuestionen sus decisiones. 2) ataque mediante aislamiento social 3) ataques a la vida privada 4) agresiones verbales como gritar o insultar, criticar permanentemente el trabajo de esa persona 5) rumores: criticar y difundir rumores contra esa persona".

En consecuencia, se desprende una clara consideración que muestra que el mobbing afecta la calidad de vida laboral, atentando contra las condiciones idóneas para realizar las actividades establecidas, hecho que tiene repercusiones en todos los aspectos de la vida del acosado moralmente tanto personal, como laboralmente. Atendiendo a estas consideraciones, resulta evidente la necesidad de analizar al mobbing como elemento causante de accidentes de trabajo, como un factor de riesgos psicosocial en las relaciones de trabajo, para describir los efectos de este acoso en la victima y poder así determinar qué elementos pueden generar accidentes de trabajo.

Desde la perspectiva teórica, esta investigación significa un aporte, pues está orientada a definir nuevas líneas de investigación que pueden generar nuevos conocimientos debido a que en esta materia del mobbing en Venezuela y Ecuador se está desarrollando abundante doctrina y legislación.

Asimismo, en el aspecto social este estudio aportará una nueva perspectiva humanística 
interesada en el bienestar del ser humano, ya que no solo abarcará un tema poco comentado, sino que pretende dar a conocer diversos aspectos que servirá de apoyo a las víctimas de acoso moral, así como a todo trabajador y/o persona que tenga interés en estudiar la materia, permitiendo que la misma sea difundida para así identificar y analizar las acciones que permitan prevenir, corregir y sancionar este fenómeno.

Con respecto al punto de vista práctico, este artículo se denota importante ya que el mismo hará posible definir y proponer lineamientos que conduzcan a la creación de unas normativas y reglamentaciones adecuadas, para el sano tratamiento del mobbing y sus diferentes efectos en la vida de los trabajadores afectados.

Por otra parte, hablando metodológicamente, resulta una contribución resaltante debido al nuevo enfoque planteado hacia un problema psicológico relacionado con el trabajo, el cual se manifiesta a través del mobbing, acoso moral, psicoterror o violencia psicológica, relacionándolo a su vez con los accidentes laborales, cuyo desarrollo será posible a través de la aplicación de métodos de investigación que permiten dar sustento científico y confiabilidad a los resultados obtenidos.

\section{Conclusiones}

El acoso psicológico no es solo un riesgo psicosocial, sino uno de los más peligrosos, puesto que la presión psicológica a la cual se ve sometido una persona sobrepasa todo nivel de tolerancia y autocontrol, esto como resultado de la destrucción de los medios de comunicación de la víctima, de su reputación personal y hasta profesional, de su humillación e incluso la pérdida de un óptimo estado de salud física.

La existencia de diversos tipos de psicoterror agrava el asunto, pues demuestra que a nivel organizacional nadie está exento de ser víctima de este fenómeno, puede ocurrir de forma ascendente, un empleado que acosa a su jefe; descendente, un empleado que es acosado por su jefe y horizontal, donde el acoso ocurre entre individuos del mismo nivel de jerarquía.

De lo que no se tiene certeza es qué impulsa un fenómeno de la magnitud del acoso moral; sin embargo, existen múltiples causas relacionadas a este, pero ninguna que justifique tal violación de los derechos humanos, que sea precisa y que pueda ayudar a determinar una forma de detenerlo o aminorar sus efectos; no obstante, se han estudiado una serie de fases que describen el proceso de acoso y las partes implicadas en dicho proceso.

Otro agravante del mobbing es que sus víctimas no tienen un perfil definido, este fenómeno puede afectar a cualquier persona, no se distingue sexo, raza o religión, cualquier trabajador puede ser víctima, sin importar su jerarquía, cuan brillante o desapercibido que sea, en la organización todos pueden ser afectados por este mal.

El mobbing como factor de riesgo psicosocial en las relaciones de trabajo es un fenómeno en incremento, que deteriora la calidad de vida de las personas acosadas moralmente y de quienes le rodean, ya que atenta directamente contra los principios básicos de derechos humanos, afectando física y psicológicamente a la víctima, los ataques psicológicos del agresor dejan imborrables 
cicatrices llevando al trabajador acosado moralmente a un estado de hipervigilancia eterna en el que desconfía de todo y de todos.

De todos los riesgos psicosociales, el mobbing es el que atípicamente presenta aspectos que indican el carácter consciente y deliberado de ocasionar un determinado daño en la víctima, siendo su agente causal otra persona que con conductas más o menos disimuladas, pero conscientes afecta psicológica y en consecuencia físicamente a su víctima.

El fin principal del acoso moral es causar intencionalmente y con premeditación un daño psicológico en la víctima, que motive a las misma a retirarse de su lugar de trabajo, es así como este fenómeno puede ser considerado fácilmente como una causal justificada de retiro, pues los estímulos negativos a los que se ve expuesta la victima vulneran de una manera incalculable sus derechos humanos. 


\section{Referencias}

Asamblea Nacional de la República Bolivariana de Venezuela (1999), Constitución de la República Bolivariana de Venezuela, con Exposición de motero, Según Gaceta Nº 5.453 Extraordinaria del 24 de marzo del 2000. Editores Hermanos Vadell C.A, Valencia Venezuela.

Asamblea Nacional de la República Bolivariana de Venezuela, Ley Orgánica del Trabajo Trabajadores y Trabajadoras, Gaceta Oficial N 6.076 Extraordinario del 7 de mayo de 2012.

Asamblea Nacional de la República Bolivariana de Venezuela (2005), Ley Orgánica de Prevención, Condiciones y Medio Ambiente de Trabajo, Gaceta Oficial № 38.236 del 26 de julio de 2005.

Barbado, P. (2004). Los derechos vulnerados por el acoso psicológico en el ámbito laboral de los poderes públicos. Jurisprudencia Argentina, 2004c, IV, 29.

Constitución de la República del Ecuador. Resolución Legislativa No. 0, publicada en Registro Oficial Suplemento 653 de 21 de diciembre del 2015.

Código Penal Ecuador. Codificación 0 Registro Oficial Suplemento 147 de 22-ene-1971 Ultima modificación: 15-feb-2012

Código del Trabajo Ecuador. Codificación 17 Registro Oficial Suplemento 167 de 16-dic-2005 Ultima modificación: 26-sep-2012

Pelekais, C., Finol, M., Neuman, N., \& Parada, J. (2012). El ABC de la investigación. Maracaibo. Venezuela.

González, M. (2005). Una nueva causal de retiro justificado del trabajo: The Mobbing (psicoterror, acoso sexual y estrés laboral) en Venezuela. Documento en línea http://www.monografias.com/trabajos43/mobbing/mobbing.shtml

Guevara, L. (2003). Tratamiento del acoso psicológico, el estrés y el burnout como accidentes de trabajo. Cuba. Marzo.

Hirigoyen, M. (2001). El acoso moral en el trabajo. Distinguir lo verdadero de lo falso [Bullying at work. Distinguish the true from the false].

Leymann, H. (1996). Contenido y Desarrollo del Acoso Grupal/moral ("Mobbing") en el Trabajo. (Traducido por Francisco Fuertes Martínez). European Journal of Work and Organizational Psicology, 5(2), 165.

Organización Internacional del Trabajo (OIT). (2006). Aumentan las nuevas formas de violencia en el trabajo, 
dice informe de la OIT. https://www.ilo.org/global/about-the-ilo/newsroom/news/WCMS 070534/lang--es/ index.htm

Organización de los Derechos Humanos (ONU). (1948). Declaración Universal de Derechos Humanos, resolución 217 A (III). https://undocs.org/es/A/RES/217(III)

Velázquez, M., \& Social, S. (2001). La respuesta jurídico legal ante el acoso moral en el trabajo o mobbing. Retirado em, 18(08), 2004.

Velásquez, M. (2002). El Marco Jurídico del acoso moral o mobbing. Documento en línea. Diario de Noticias. www.ugt.es/actualidad/mobbing.pdf 


\section{Copyright (c) 2020 Juan Gerardo Ávila Urdaneta}

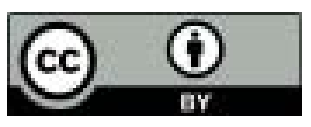

Este texto está protegido bajo una licencia internacional Creative Commons 4.0.

Usted es libre para Compartir-copiar y redistribuir el material en cualquier medio o formato - y Adaptar el documento - remezclar, transformar y crear a partir del material-para cualquier propósito, incluso para fines comerciales, siempre que cumpla las condiciones de Atribución. Usted debe dar crédito a la obra original de manera adecuada, proporcionar un enlace a la licencia, e indicar si se han realizado cambios. Puede hacerlo en cualquier forma razonable, pero no de forma tal que sugiera que tiene el apoyo del licenciante o lo recibe por el uso que hace de la obra.

\section{$\underline{\text { Resumen de licencia - Texto completo de la licencia }}$}

